\title{
1 The Circadian Clock regulates RPE-mediated Lactate Transport via SLC16A1 (MCT1)
}

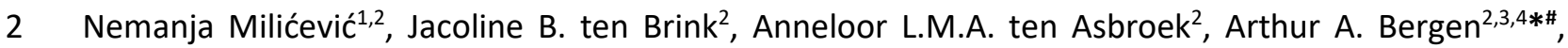
3 Marie-Paule Felder-Schmittbuhl ${ }^{1 *}$

4

$5 \quad{ }^{1}$ Centre National de la Recherche Scientifique, Université de Strasbourg, Institut des Neurosciences

6 Cellulaires et Intégratives, 5 rue Blaise Pascal, F-67000 Strasbourg, France

$7 \quad{ }^{2}$ Department of Clinical Genetics, Amsterdam UMC, University of Amsterdam, Meibergdreef 9, 1105

$8 \quad A Z$, Amsterdam, the Netherlands

$9{ }^{3}$ Department of Ophthalmology, Amsterdam UMC, University of Amsterdam, Meibergdreef 9, 1105

$10 \mathrm{AZ}$, Amsterdam, the Netherlands

$11{ }^{4}$ Netherlands Institute for Neuroscience (NIN-KNAW), Meibergdreef 47, 1105 BA, Amsterdam, the 12 Netherlands

Highlights

The SLC2A1 and SLC16A1 transporter genes are highly expressed in ARPE-19 cells. RPE monolayer organization is necessary for rhythmic transporter expression. POS-incubation affects transporter expression in a time-dependent manner. RPE-mediated transport is rhythmic for lactate, but not for glucose.

\section{Abstract}

Multiple retinal cells were shown to harbor a circadian oscillator, including retinal pigment epithelial cells (RPE). However, little is known about the functions that are regulated by the RPE clock. The aim of this study was to investigate whether the circadian clock in the RPE regulates the transport of glucose and its glycolytic metabolic by-product - lactate. To that end, we first characterized the mRNA expression profile of glucose and monocarboxylate transporters in ARPE-19 cells. We found that SLC2A1 and SLC16A1 were, respectively, the most abundantly expressed glucose and lactate (monocarboxylate) transporters. We further observed that the protein products of SLC2A1 (encoding GLUT1) and SLC16A1 (encoding MCT1) localize on the apical membrane of ARPE-19 monolayers. In a 
monolayers, but not in dispersed cells, suggesting that monolayer cellular organization is necessary for rhythmic regulation of these transporters. In these monolayers, we found that MCT1 proteins varied over time in contrast to GLUT1 proteins which did not vary over time. Spectrophotometric measurements of supernatants sampled from ARPE-19 monolayer cultures revealed that glucose concentrations did not significantly differ between apical (Api) supernatants and basolateral (BL) ones. In addition, we did not find rhythms in Api or BL glucose concentrations. Conversely, we found higher lactate concentrations in Api supernatants than BL ones. Further, we found that Api lactate concentrations were rhythmic. Pearson's $r$ revealed that the concentration gradients (Api - BL) of glucose and lactate correlated with the gene expression of respective SLC2A1 and SLC16A1 transporters. Incubation with photoreceptor outer segments (POS) affected the mRNA expression of SLC16A1 and SLC2A1 in ARPE-19 monolayers in a time-dependent manner, thus suggesting that the retina might modulate the RPE clock-controlled expression of transporters via interactions with POS. In conclusion, this work provides evidence that the transport of lactate is regulated by the circadian clock in the RPE.

Keywords: Retinal Pigment Epithelium, Glucose, Lactate, Epithelial Transport, Circadian Clock, Retina

\section{Introduction}

The mammalian retina contains a complete circadian clock system which regulates numerous physiological, biochemical and cellular processes within the eye (McMahon et al., 2014). These include melatonin synthesis, dopamine release, photoreceptor disc shedding and regulation of extracellular pH (McMahon et al., 2014). Virtually all retinal cells display cyclic functions, including photoreceptors (Tosini et al., 2007) as well as the adjacent monolayer of retinal pigment epithelial (RPE) cells (Baba et al., 2010). The existence of a self-sustained RPE clock is a relatively recent discovery (Baba et al., 2010). Earlier work demonstrated that cultured human RPE cells are capable of generating rhythms of clock gene transcription and adenylyl-cyclase activity (Pavan et al., 2006; Yoshikawa et al., 2008). While recent studies investigated various mechanisms of regulation of the RPE clock (Baba et al., 2017; Ikarashi et al., 2017; Morioka et al., 2018; Milićević et al., 2019), little is known about processes and functions it regulates.

The retina is among the most energy demanding tissues in the human body (Ames et al., 1992; Anderson and Saltzman, 1964; Yu and Cringle, 2001) with the photoreceptors being the most metabolically active cells in the retina (Narayan et al., 2017). Photoreceptors metabolize glucose via 
glycolysis and oxidative phosphorylation (Ames et al., 1992; Narayan et al., 2017). Glycolysis is considered as an energy-inefficient metabolic pathway as it yields 2 ATP/glucose and generates lactate as a metabolic byproduct. In contrast, oxidative phosphorylation produces 34 ATP/glucose (Ng et al., 2015). In photoreceptors, glucose metabolism exhibits day/night variations, in which glycolysis occurs primarily during light exposure and oxidative phosphorylation in darkness (Lin et al., 2015). This shift creates considerable disparities in daily photoreceptor glucose demand and production of subretinal lactate as a metabolic byproduct of glycolysis.

To meet the metabolic needs of the retina, the RPE supplies glucose and removes subretinal lactate via transporters expressed on the RPE (Adijanto and Philp, 2012). On the basolateral side of the RPE, the GLUT1 transporters move glucose from the blood in choriocapillaris through the RPE cytoplasm. Glucose is then transferred via apically expressed GLUT1 to the subretinal space. Lactate is removed from the subretinal space via apical monocarboxylate transporter (MCT) 1, partially metabolized within the RPE and the remaining lactate is secreted to the choriocapillaris via basolaterally expressed MCT3 (Adijanto and Philp, 2012). Considering that the RPE harbors a circadian oscillator (Baba et al., 2017; Baba et al., 2010; Baba and Tosini, 2018), we hypothesize that the RPE circadian clock meets the daily metabolic needs of photoreceptors via rhythmic expression of glucose and monocarboxylate transporters.

In this study, we characterized the mRNA expression profile of glucose and monocarboxylate transporters in an ARPE-19 cell culture model and the protein localization of the most abundantly expressed transporters GLUT1 and MCT1. We found rhythmicity of mRNA expression of SLC2A1 and SLC16A1 transporters as well as MCT1 proteins in monolayer but not in dispersed cultures. We probed whether the clock mediated regulation of transporter mRNA affects the glucose and lactate translocation by measuring glucose and lactate concentrations from sampled supernatants from our ARPE-19 monolayers. Finally, by incubating ARPE-19 monolayers with POS, we show that photoreceptors might affect clock-controlled mRNA expression of transporters via POS shedding.

\section{Materials and methods}

\subsection{Cell culture}

Culturing of ARPE-19 cells was performed essentially as described in (Milićević et al., 2019). In brief, ARPE-19 cells (ATCC, Manassas, VA, USA) were cultured in Dulbecco's Modified Eagle's Medium: 
Nutrient Mixture F-12 with L-glutamine and Hepes (DMEM/F12, Gibco life technologies, Carlsbad, CA, USA) suplemented with $10 \%$ heat-inactivated fetal calf serum (FCS) (Sigma-Aldrich, St. Louis, MO, USA) and $1 \%$ penicillin/streptomycin (PS) (Gibco BRL, Grand Island, NY, USA) at $37^{\circ} \mathrm{C}$ and $5 \% \mathrm{CO}_{2}$. After 5 passages, the cells were either grown as monolayers or as dispersed cells. Dispersed cells were plated on 12-well culture plates (\#3513 Costar, Kennebunk, ME, USA) at a density of $1.6 \times 10^{5}$ cells $/ \mathrm{cm}^{2}$ in a volume of $1.5 \mathrm{ml}$ and cultured for 4 days.

Monolayers were grown by plating on Matrigel-coated (160 $\mu \mathrm{l}$ of 1:40 diluted GFR Matrigel, Corning, NY, USA) transwell filters (\#3460 Costar, Kennebunk, ME, USA, 12-well plate, $12 \mathrm{~mm}$ diameter, $0.4 \mu \mathrm{m}$ pore size). The seeding density was $1.6 \times 10^{5} \mathrm{cells} / \mathrm{cm}^{2}$ in a total volume of $0.5 \mathrm{ml} /$ filter. Monolayers were cultured using DMEM/F12 medium supplemented with 1\% FCS and PS for 28 days. Growth and morphology was monitered using microscopy. Before applying lysis buffer (PCR and WES ${ }^{\mathrm{TM}}$ experiments), matrigel was first removed from the cells by incubating in Cell Recovery solution (Corning, Bedford, MA, USA) for 30 min at $4^{\circ} \mathrm{C}$.

Synchronization of cells was performed by applying 50\% FCS in DMEM/F12 between time-points 0-2h ("serum-shock"), followed by a medium change using serum-free DMEM/F12 as described previously (Balsalobre et al., 1998).

We measured transepithelial electrical resistance (TEER) of monolayer samples prior to experiments using an ohmmeter (Millicell ERS-2, Billerica, MA, USA). We performed a background correction of TEER measurements by subtracting the value of a Matrigel coated filter without cells from TEER measurements with cells. The resulting values were multiplied by transwell filter area. We chose TEER of $20 \Omega \mathrm{cm}^{2}$ as a threshold value for intact monolayers.

\subsection{RNA isolation, CDNA synthesis and mRNA quantification}

We extracted total RNA using the RNeasy mini kit (Qiagen, Valencia, CA, USA) according to the manufacturer's protocol. We used $50 \mathrm{ng}$ of total RNA to generate complementary DNA with oligo(d.T) primed reactions using Superscript III reverse transcriptase (Life technologies, Waltham, MA, USA). The synthesized CDNA was amplified with transcript-specific, intron-spanning primers. We used PCR programs optimized for transporter mRNA expression profiling (Fig. 1) and quantification (Fig. 2, Table 2 and 5). Table 1 shows the sequences of used primers. After electrophoresis on $2 \%$ agarose gels, PCR products were imaged using the FujiFilm LAS300 (Tokyo, Japan) and quantified using Aida image 
analyzer v4.26. Normalization was performed by division with the signal obtained from the PCR product of the reference gene $E F 1 \alpha$ and by using average expression values within an experiment as a calibrator between experiments.

\begin{tabular}{|c|c|c|}
\hline Gene & Forward Primer & Reverse Primer \\
\hline \multicolumn{3}{|c|}{ Glucose transporter genes } \\
\hline SLC2A1 & CACTGTCGTGTCGCTGTTTG & TCCGGCCTTTAGTCTCAGGA \\
\hline$S L C 2 A 2$ & CAGTCAAGGACCACGTCCTG & CAGTCTCTGTAGCTCCTAGG \\
\hline SLC2A3 & ACTTACTCCCGGAACGCTGG & TGGCTGGTAGAGCCCACTTC \\
\hline SLC2A4 & CATGGGTTTCCAGTATGTTGC & GGTGGAAGGCAGCTGAGATC \\
\hline SLC2A5 & CCACGTCATAGGACATGCC & TGGTGAGGAGGCAGATCACG \\
\hline \multicolumn{3}{|c|}{ Monocarboxylate transporter genes } \\
\hline SLC16A1 & TGTTGACATGGTAGCCCGAC & TGAGCCGACCTAAAAGTGGT \\
\hline SLC16A7 & AACTCTCATGGACCTCGTGG & GCCAATGAGCAGCCACACGC \\
\hline SLC16A8 & GCCTCTCCTACGGCATGGTG & GGCGCTGAGCACСTCCAGG \\
\hline SLC16A4 & TGCCTGTACTGGTTGATCTGT & TGCGATGTGTTTCTTTCAAGC \\
\hline SLC16A5 & $\begin{array}{r}\text { TCTTCСTCATCTCAGCTGCC } \\
\text { Clock genes }\end{array}$ & САCTCATGGCTGCTCCTGGA \\
\hline$R E V-E R B \alpha$ & TAAGCCGCACCACCTACAGC & GGCCAGAGGCTCATCTTGGA \\
\hline \multicolumn{3}{|c|}{ Reference gene } \\
\hline$E F 1 \alpha$ & AAATAAGCGCCGGCTATGCC & CAAAGCGACCCAAAGGTGGAT \\
\hline
\end{tabular}

\subsection{Western Blotting}

ARPE-19 monolayers were "serum-shocked" ( $t=0-2 \mathrm{~h})$, given serum-free medium $(\mathrm{t}=2 \mathrm{~h})$ and harvested at $t=12 \mathrm{~h}$ and $\mathrm{t}=24 \mathrm{~h}$. Matrigel was removed, and cells were lysed with RIPA buffer ( $10 \mathrm{mM} \mathrm{Tris} / \mathrm{HCl} \mathrm{pH}$ 8, $140 \mathrm{mM} \mathrm{NaCl}, 1 \mathrm{mM}$ EDTA, $0.5 \mathrm{mM}$ EGTA, 0.1\% SDS, 0.1\% Na-deoxycholate, 1\% Triton X-100) supplemented with phosphatase (PhosSTOP, Roche, Mannheim, Germany) and protease inhibitors (cOmplete, Roche). We used a modified Lowry assay (DC protein assay, Bio-Rad laboratories, USA) to determine total protein concentrations. Concentrations varied between 0.55 and $0.76 \mathrm{mg} / \mathrm{ml}$. Protein detection and quantification was performed using the capillary-based automated WES ${ }^{\text {TM }}$ Simple Western (ProteinSimple, San Jose, USA). All procedures were performed according to the manufacturer's instructions (Harris, 2015) with the following modifications: For GLUT1 detection and quantification, we prepared samples using $3.5 \mu \mathrm{l}$ of protein lysates, $0.5 \mu \mathrm{l}$ of $0.1 \mathrm{X}$ sample buffer and $1 \mu \mathrm{l}$ of fluorescent marker. Samples were heated at $55^{\circ} \mathrm{C}$ for $15 \mathrm{~min}$ and loaded into each WES ${ }^{\mathrm{TM}}$ plate 
well. For MCT1 detection and quantification, we used $3 \mu$ of protein lysates, $1 \mu \mathrm{l}$ of $1 \mathrm{X}$ sample buffer supplemented with $2.5 \% \beta$-mercaptoethanol and $1 \mu$ l of fluorescent marker. Samples were heated at $37^{\circ} \mathrm{C}$ for $30 \mathrm{~min}$ and loaded into each WES ${ }^{\mathrm{TM}}$ plate well. We used the following primary antibodies: GLUT1 (R\&D Systems, $40 \mu \mathrm{g} / \mathrm{ml}$, MAB14181) and MCT1 (Abcam, 1:15, AB90582). After WESTM quantification, we normalized the protein expression profiles of GLUT1 and MCT1 with total protein concentrations of the respective sample lysate, as previously described (Milićević et al., 2019)

\subsection{Photoreceptor Outer Segment isolation}

Bovine eyes were obtained from the slaughterhouse. After cutting the eyes open, we scrapped the retinas from posterior eye cups. Retinas were homogenized in $42 \%$ sucrose, $1 \mathrm{M} \mathrm{NaCl}, 0.1 \mathrm{M} \mathrm{MgCl} 2$ and $1 \mathrm{M}$ Tris-acetate. The supernatant was centrifuged, filtered through a sterile gauze and diluted in 2 volumes of $0.01 \mathrm{M}$ Tris-acetate. POS were separated in a sucrose gradient by ultracentrifugation (Optima ultracentrifuge, Beckman Coulter, Inc., Brea, CA) at 25,000 RPM for 40 min at $4^{\circ} \mathrm{C}$. POS were recovered in $0.01 \mathrm{M}$ Tris-acetate, ultracentrifuged at 20,000 RPM for $30 \mathrm{~min}$ at $4^{\circ} \mathrm{C}$, and disolved in DMEM:F12 medium. The number of POS particles / $\mathrm{ml}$ was determined by manual counting using a haemocytometer.

\subsection{Photoreceptor Outer Segment incubation assay}

After synchronization of ARPE-19 monolayers $(t=2 h)$, serum-containing medium was removed and $0.4 \mathrm{ml}$ and $1 \mathrm{ml}$ of serum-free medium was administered to apical and basolateral compartments, respectively. At the intervention time-point (either $\mathrm{t}=9 \mathrm{~h}, 15 \mathrm{~h}, 21 \mathrm{~h}, 27 \mathrm{~h}$ or $33 \mathrm{~h}$ ) $100 \mu \mathrm{l}$ of medium or POS was administered. Cells were incubated with $1.2 \times 10^{7}$ POS particles for $3 \mathrm{~h}$ (or $6 \mathrm{~h}$, see Supplementary file) at $37^{\circ} \mathrm{C}$ in $5 \% \mathrm{CO}_{2}$. After a $3 \mathrm{~h}$ incubation, the cells were washed, harvested, cleaned from Matrigel and lyzed.

\subsection{Immunocytochemistry and imaging}

Cells were fixed with $4 \%$ paraformaldehyde for $10 \mathrm{~min}$ at RT. Transwell filters with cells were cut and mounted on poly-L-lysine coated glass coverslips. Blocking was performed using $1 \% \mathrm{BSA}, 10 \%$ normal donkey serum, 0.2\% Triton X-100 in PBS. Incubation with primary antibodies (MCT1, 1:250, Abcam, AB90582; GLUT1, 1:250, ThermoFisher, MA5-11315) was performed for $16 \mathrm{~h}$ at $4{ }^{\circ} \mathrm{C}$. Incubation with 
Cy3 conjugated donkey anti-mouse secondary antibodies (Jackson ImmunoResearch, 715-165-150, USA) was performed for 60 min at RT. Samples were mounted with Vectashield antifade medium with DAPI (Vector laboratories, Burlingane, CA, USA). Confocal stacks were acquired with a Leica TSC SP-8 mounted on a Leica DMI6000 inverted microscope using a HC Plan Apochromat x63 objective.

\subsection{Biochemical analysis}

192

After performing the "serum-shock" synchronization on ARPE-19 monolayers (Balsalobre et al., 1998), we applied exactly $0.5 \mathrm{ml}$ of serum-free medium apically and $1 \mathrm{ml}$ basolaterally. Apical and basolateral supernatants were sampled immediately before cell harvesting (for PCRs). An aliquot of $100 \mu \mathrm{l}$ was submitted to biochemical analysis (Cobas 8000 ISE900 Chemistry Analyzer, Roche Diagnostics, Rotkreuz, Switzerland) at the Department of Clinical Chemistry, Amsterdam UMC. Glucose (kit: GLUC3 glucose HK gen.3, Roche 05168791190) and lactate concentrations (kit: LACT2 Lactate Gen.2, Roche 05171881190) were measured according to manufacturer's instructions (Roche Diagnostics, Rotkreuz, Switzerland).

201

\subsection{Data analysis}

203

\subsubsection{Analysis of glucose and lactate concentrations.}

205

206

Data analysis was performed on raw and detrended glucose and lactate concentrations. To remove 207 the trend (continuous decrease/increase in measurements) of glucose and lactate concentrations, we first performed linear regression on the raw glucose $\left(p<0.0001, r^{2}=0.84, y=-0.1196 \cdot t+17.4\right)$ and lactate data $\left(p<0.0001, r^{2}=0.84, y=0.2263 \cdot t+0.4362\right)$. To detrend the data, we subtracted the respective calculated y values from each glucose and lactate measurement. To remove negative y values after detrending, the minimum y value was set to zero. In that regard, we added, respectively, 0.7296 and 1.5674 to each detrended glucose and lactate measurement. Further analysis was performed on detrended data. Concentration gradients were calculated by subtracting basolateral from apical concentrations.

Data were obtained from at least 3 biological replicates and expressed as mean $\pm S E M$. Plots were generated using GraphPad Prism software (La Jolla, CA, USA) or SigmaPlot (Systat Software, San Jose, 

expression profiles were determined by nonlinear least squares fitting to a sine wave function using

222 SigmaPlot: $y=y_{0}+c \cdot \cos [2 \pi(t-\varphi) / \tau]$, where $\tau$ represents period, $\varphi$ acrophase and $c$ amplitude. The 223 function featured the following contraints: $20 \mathrm{~h}<\tau<28 \mathrm{~h} ; \varphi<\tau$ and $c>0$. Where indicated analysis was 224 performed with a 2-way ANOVA followed by Holm-Sidak's post-hoc tests or 1-way ANOVA. 225 Correlations between gene expression and solute concentration gradients were performed using Pearson's r.

227

\section{Results}

229

\subsection{Glucose and lactate transporter expression profile in ARPE-19 cell cultures}

We first characterized the mRNA expression profile of glucose transporters in ARPE-19 cell cultures. RT-PCR analysis revealed that in both ARPE-19 dispersed cells and monolayers, the expression of SLC2A1 mRNA was considerably high, while expression of other glucose transporters was remarkably low (SLC2A5) or below detection (SLC2A2-4) (Fig. 1a).

Next, we examined the monocarboxylate transporter mRNA expression profile of ARPE-19 cells (Fig. 1b). RT-PCR analysis revealed detectable expression of SLC16A5 and SLC16A7, but not of SLC16A4 and SLC16A8, in both ARPE-19 cultures. Of all monocarboxylate transporter genes, the mRNA expression of SLC16A1 was the highest in our ARPE-19 cultures.

241 Finally, we aimed to find the localization of proteins encoded by the most abundant glucose (SLC2A1) 242 and monocarboxylate (SLC16A1) transporters in ARPE-19 monolayers, and compare these with the in 243 vivo RPE situation. Confocal microscopy revealed that GLUT1 (Fig. 1c) and MCT1 (Fig. 1d) localized on 244 the apical membrane of ARPE-19 monolayers. Therefore, our ARPE-19 monolayers displayed MCT1 245 localization similar to in vivo RPE (Philp et al., 1998). However, they lacked basolateral expression of GLUT1 (Sugasawa et al., 1994). 


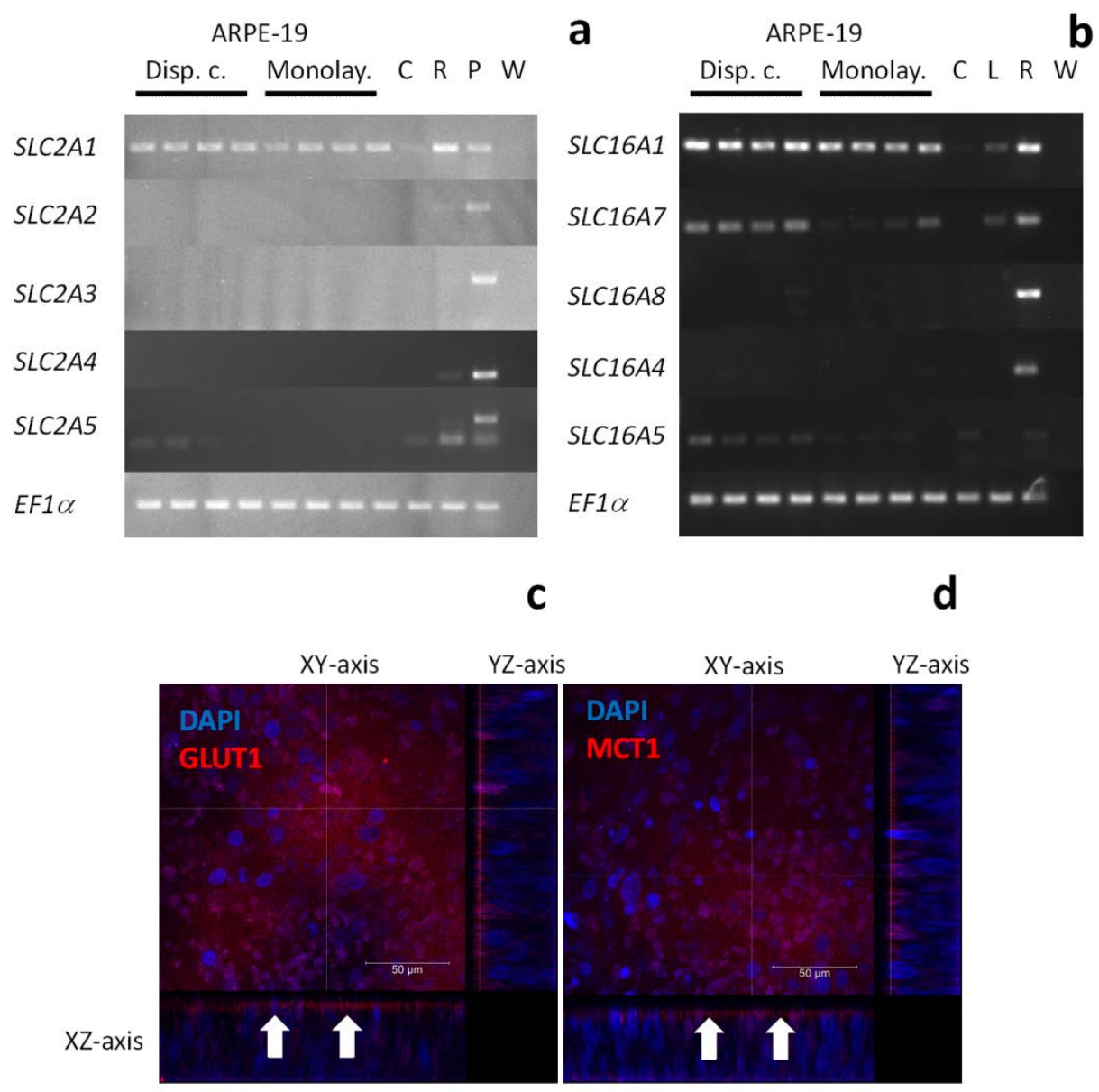

248 Figure 1. Expression profile of glucose and monocarboxylate transporters in ARPE-19 cell cultures. (a) 249 The positive controls used in RT-PCR analysis were: cortex (C), retina (R), placenta (P) and liver (L) cDNA. 250 W - water. The ARPE-19 dispersed cells (Disp. c.) and monolayer (Monolay.) samples were obtained 251 from 4 independent cultures. These cells expressed the SLC2A1 glucose transporter. Gene expression 252 of SLC2A5 was very low and SLC2A2-4 mRNA was not detectable in ARPE-19 cells. (b) SLC16A1-8 253 transporter expression profile in ARPE-19 cells. Immunohistochemical localization of (c) GLUT1 and (d) 254 MCT1 in ARPE-19 monolayers. Nuclei were labeled with DAPI. Confocal microscopy revealed that 255 GLUT1 and MCT1 localized on the apical membrane of ARPE-19 monolayers (white arrows).

3.2. Expression profile of glucose and monocarboxylate transporter genes is rhythmic in monolayer, but not in dispersed ARPE-19 cell cultures

Our previous work indicated that cellular organization of the RPE affects circadian parameters of clock and clock-controlled gene expression in ARPE-19 cultures (Milićević et al., 2019). To investigate 
whether the ARPE-19 monolayers have enhanced rhythmicity of transporter gene expression with respect to dispersed ARPE-19 cells, we performed a series of RT-PCR analyses on mRNA from both cultures. We synchronized ARPE-19 cells using the serum-shock procedure by giving them $50 \%$ serum $(t=0-2 h)$, then serum-free medium $(t=2 h)$. We harvested cells at $3 h$ intervals for $54 h$ and we characterized the circadian expression profile of SLC2A1 and SLC16A1 by RT-PCR.

The clock genes REV-ERB $\alpha$ and PER2 were shown to be among the most rhythmically expressed in ARPE-19 dispersed and monolayer cultures (Milićević et al., 2019). Thus, in this study, we performed a RT-PCR analysis on these genes to confirm the rhythmicity of our ARPE-19 cultures. As expected, RTPCR analysis showed that REV-ERB $\alpha$ and PER2 were indeed rhythmic in both dispersed and monolayer cultures (Fig. 2; Table 2). Dispersed ARPE-19 cell cultures did not show rhythmic mRNA expression of SLC2A1 (Fig. 2; Table 2) and SLC16A1 (Fig. 2; Table 2). In contrast, ARPE-19 monolayers did show rhythmic mRNA expression of SLC2A1 (Fig. 2; Table 2) and SLC16A1 (Fig. 2; Table 2). These results suggest that the RPE monolayer cellular organization is necessary for rhythmic mRNA expression of SLC2A1 and SLC16A1 transporters.

Dispersed cell culture

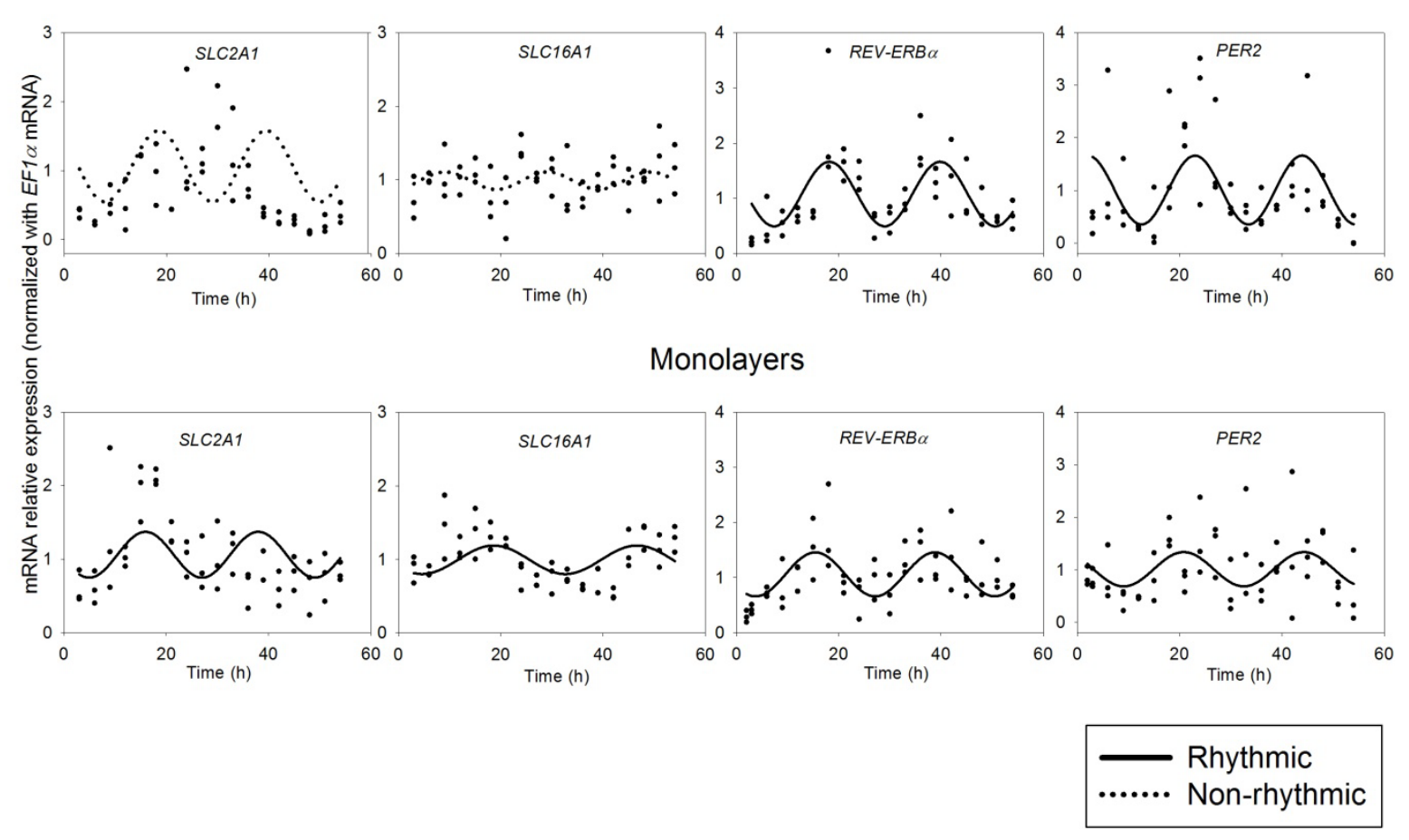

279 Figure 2. The monolayer cellular organization of the ARPE-19 is necessary for rhythmic mRNA 280 expression of the SLC2A1 glucose and SLC16A1 monocarboxylate transporters. Time is represented on 281 the $x$-axis, relative mRNA expression is on the $y$-axis. Dispersed (Top) and monolayer (Below) ARPE-19 
cells were cultured. Cells were synchronized using $50 \%$ serum $(t=0-2 h)$, then the medium was changed to serum-free medium $(t=2 \mathrm{~h}$ ), followed by cell harvest at $3 \mathrm{~h}$ intervals and RT-PCR analysis. Traces represent the best fitted sinusoidal regressions supporting rhythmic mRNA expression (dashed lines when non-rhythmic expression).

\begin{tabular}{|c|c|c|c|c|c|c|}
\hline \multicolumn{5}{|c|}{ Non-linear regression analysis } & \multicolumn{2}{|c|}{ 1-way ANOVA } \\
\hline Gene & $P$ value & Period (h) & Acrophase (h) & Amplitude & $P$ value & F value \\
\hline \multicolumn{7}{|c|}{ Dispersed ARPE-19 cells } \\
\hline \multicolumn{7}{|c|}{ Transporter genes } \\
\hline$S L C 2 A 1$ & 0.3818 & $21.00 \pm 2.38$ & $18.51 \pm 2.10$ & $0.52 \pm 0.29$ & 0.0033 & 2,93 \\
\hline SLC16A1 & 0.2827 & $20.00 \pm 1.85$ & $9.10 \pm 2.33$ & $0.12 \pm 0.06$ & 0.2076 & 1.37 \\
\hline \multicolumn{7}{|c|}{ Clock genes } \\
\hline$R E V-E R B \alpha$ & $<0.0001$ & $21.64 \pm 0.81$ & $18.18 \pm 0.69$ & $0.58 \pm 0.10$ & $<0.0001$ & 5.14 \\
\hline PER2 & 0.0010 & $20.95 \pm 1.12$ & $2.05 \pm 1.61$ & $0.66 \pm 0.15$ & 0.0170 & 2.31 \\
\hline \multicolumn{7}{|c|}{ ARPE-19 monolayers } \\
\hline \multicolumn{7}{|c|}{ Transporter genes } \\
\hline$S L C 2 A 1$ & 0.0218 & $22.05 \pm 1.54$ & $15.94 \pm 1.46$ & $0.31 \pm 0.10$ & $<0.0001$ & 4.49 \\
\hline SLC16A1 & 0.0198 & $28.00 \pm 2.60$ & $18.59 \pm 1.78$ & $0.19 \pm 0.06$ & $<0.0001$ & 6.18 \\
\hline \multicolumn{7}{|c|}{ Clock genes } \\
\hline$R E V-E R B \alpha$ & $<0.0001$ & $23.37 \pm 1.20$ & $15.44 \pm 1.02$ & $0.40 \pm 0.08$ & 0.0024 & 2.97 \\
\hline PER2 & 0.0475 & $23.48 \pm 1.63$ & $20.80 \pm 1.25$ & $0.33 \pm 0.11$ & 0.1117 & 1.59 \\
\hline
\end{tabular}

Table 2. Statistical analysis of transporter and clock gene rhythmicity in ARPE-19 dispersed and monolayer cultures. Data were fitted to the equation $y=y_{0}+c \cdot \cos [2 \pi(t-\varphi) / \tau]$ by non-linear regression. Bold values represent $p<0.05$

\subsection{Do transporter proteins show temporal variations in ARPE-19 monolayers?}

Considering that the monolayer cellular organization is necessary for rhythmic expression of transporter mRNA, we hypothesized that in monolayers, transporter proteins also show temporal variations. To test this hypothesis, we synchronized ARPE-19 monolayers $(\mathrm{t}=0-2 \mathrm{~h})$ and harvested these cells at 2 time-points $\left(\mathrm{t}=12 \mathrm{~h}\right.$ and $\mathrm{t}=24 \mathrm{~h}$ ). Using the WES ${ }^{\mathrm{TM}}$ capillary based protein quantification technique, we found that GLUT1 protein levels did not show statistically significant differences 
306

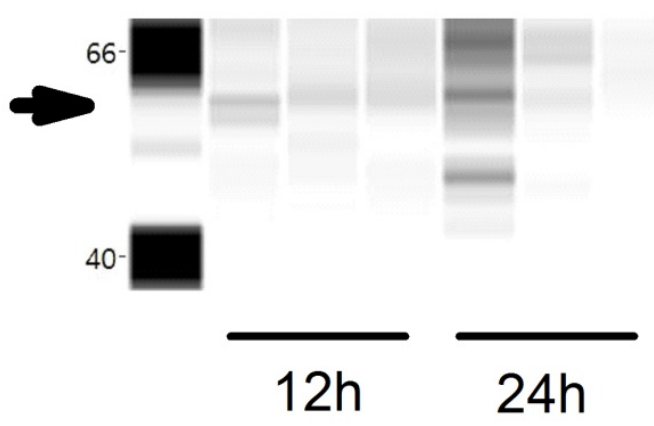

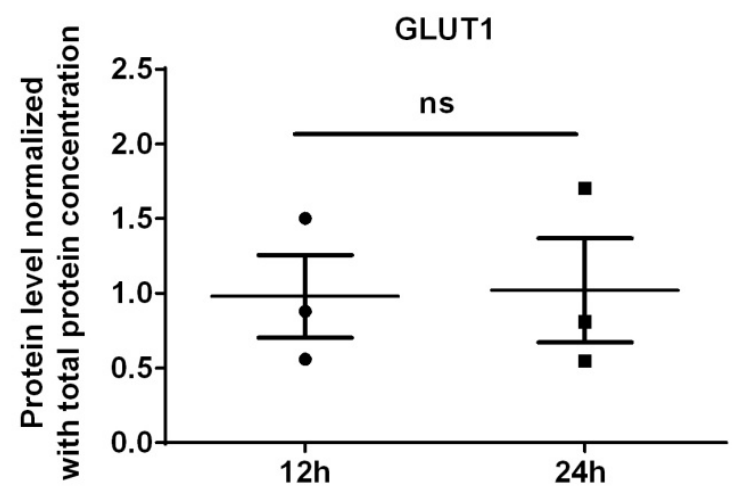
to $S L C 2 A 1$ which shows rhythms only on the mRNA level.$$
\text { to SLC2A1 which shows rhythms only on the mRNA level. }
$$

between $\mathrm{t}=12 \mathrm{~h}$ and $\mathrm{t}=24 \mathrm{~h}$ (Student's $t$-test, $\mathrm{p}=0.94$ ). Conversely, differences in MCT1 protein levels between these 2 time-points were significantly different (Student's $t$-test, $p=0.01$ ). These results suggest that in ARPE-19 monolayers, SLC16A1 rhythmicity is translated to the protein level, in contrast

Figure 3. Time affects the protein expression of MCT1 but not GLUT1 in ARPE-19 monolayers. ARPE-19 monolayers were synchronized by "serum-shock" ( $t=0-2 h)$, given serum-free medium $(t=2 h)$ and harvested at $t=12 \mathrm{~h}$ and $24 \mathrm{~h}$. (Top) Quantification of proteins using WES ${ }^{\mathrm{TM}}$ revealed no statistically significant differences in GLUT1 proteins between $t=12 \mathrm{~h}$ and $\mathrm{t}=24 \mathrm{~h}$ (Student's $t$-test, $\mathrm{p}=0.94$ ). In contrast, MCT1 protein levels differed between $t=12 \mathrm{~h}$ and $\mathrm{t}=24 \mathrm{~h}$ (Student's $t$-test, $\mathrm{p}=0.01$ ). Individual values, means and SEM are plotted. (Bottom) Cropped digitally generated WES ${ }^{\mathrm{TM}}$ images are shown (uncropped are provided in Figs S2-S3). GLUT1 was detected at $61 \mathrm{kDa}$ and MCT at 47-48 kDa. 
Since we observed rhythmic mRNA expression of the main retinal glucose transporter SLC2A1 and

319 lactate transporter SLC16A1, we wanted to determine whether this rhythm also has functional 320 consequences. We opted for an indirect approach by measuring remaining glucose and accumulating 321 lactate in ARPE-19 monolayer supernatants sampled from apical (Api) and basolateral (BL) compartments.

323

ARPE-19 monolayers were synchronized using the serum-shock procedure as previously described. We sampled Api and BL supernatants at $6 \mathrm{~h}$ intervals for $30 \mathrm{~h}$. As expected, a two-way ANOVA analysis revealed that glucose concentrations continuously decreased over sampling time (Fig. $4 a, F(4,20)=$ 29.54, $p<0.0001)$, but were not affected by compartment $(F(1,20)=1.54, p=0.23)$ or time $x$ compartment $(F(4,20)=0.98, p=0.44)$. To determine whether the concentration of glucose oscillated in supernatants, we performed non-linear regression on detrended data (Fig. 4c). We did not find rhythms in Api ( $p=0.11)$ or BL $(p=0.52)$ glucose concentrations. We then calculated glucose gradients for each time-point by subtracting Api - BL glucose concentrations. We found that Pearson's linear regression revealed a significant negative relationship between the relative mRNA expression of SLC2A1 and calculated glucose gradients $(r=-0.60, p<0.05)$. Taken together, these results suggest that RPE-mediated glucose transport is not rhythmic.

We then measured lactate concentrations from $A p i$ and $B L$ supernatants from our time-course experiment. Two-way ANOVA testing showed that lactate concentrations were significantly increased over sampling time (Fig. $4 b, F(4,20)=317, p<0.0001$ ) and were affected by sampling compartment $(F(1,20)=315.5, p<0.0001)$ and had a tendency for time $x$ compartment $(F(4,20)=2.50, p=0.08)$. These results suggest that lactate was either preferentially secreted in, or translocated to the Api side of ARPE-19 monolayers. Non-linear regression analysis on detrended data showed that Api lactate was rhythmic ( $c=0.78 \pm 0.15 ; \varphi=12.24 \pm 0.80 h ; \tau=26.54 \pm 1.99 \mathrm{~h}$ and $p<0.01$ ) and BL showed a tendency $(p=0.06)$. To confirm if the lactate transfer rate is correlated with the mRNA of the SLC16A1 transporter we performed Pearson's $r$ analysis. Pearson's linear regression showed a significant relationship between the relative gene expression of SLC16A1 and lactate concentration gradients $(r=$ $0.68, p<0.05)$. In summary, these results suggest that the circadian clock regulates the translocation of lactate. 


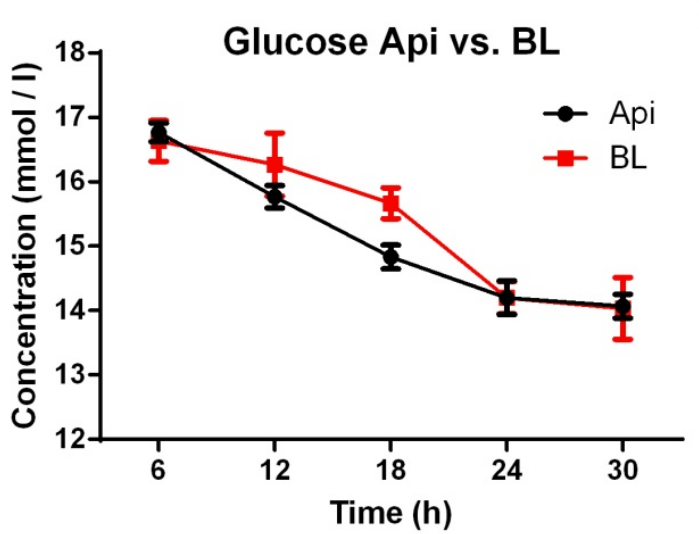

a
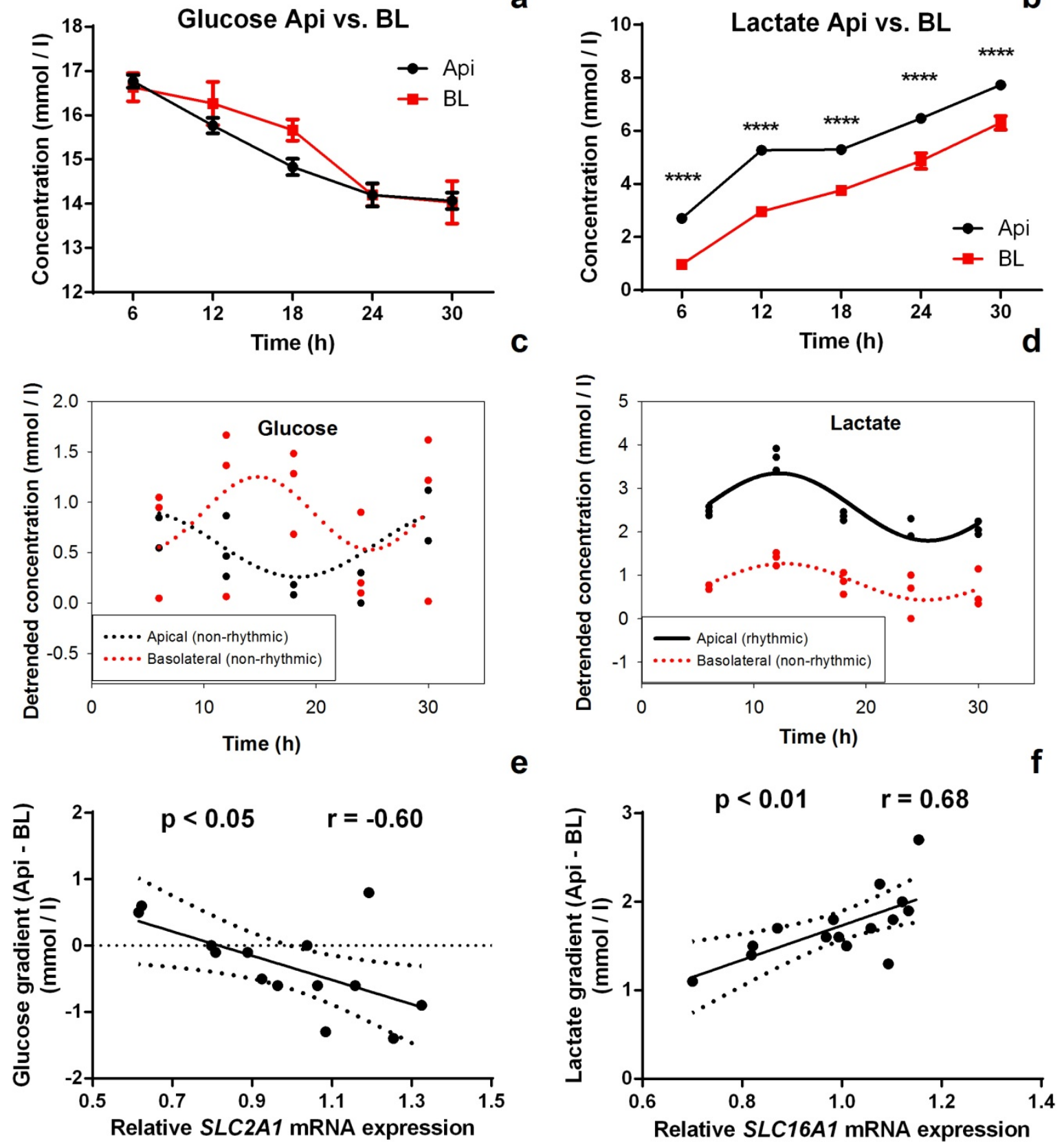

Figure 4. Temporal analysis of supernatant glucose and lactate concentrations of ARPE-19 monolayers. Apical (Api) and basolateral (BL) supernatants were sampled from ARPE-19 monolayers at $6 \mathrm{~h}$ intervals.

351 Cells were synchronized using medium supplemented with $50 \%$ fetal calf-serum at $\mathrm{t}=0-2 \mathrm{~h}$ followed by 352 a medium change at $\mathrm{t}=2 \mathrm{~h}$. (a) Glucose and (b) lactate concentrations were measured from Api and BL supernatants. Non-linear regression was performed on detrended (c) glucose and (d) lactate concentrations. Glucose and lactate concentration gradients were calculated by subtracting BL concentrations of respective solutes from Api ones. Results of Pearson's $r$ correlation revealed a 
relationship between (e) glucose and (f) lactate transporter mRNA and concentration gradients of respective solutes. Values are plotted on graphs or shown as means $\pm \mathrm{SEM}, \mathrm{n}=3$ / time-point / treatment. ${ }^{* \star * *} p<0.0001$

\subsection{Incubation with POS regulates the mRNA expression of the SLC2A1 and SLC16A1 transporters.}

Our previous experiments showed that SLC2A1 and SLC16A1 exhibit rhythmic mRNA expression in the RPE (Fig. 2). Considering that the RPE and photoreceptors lie in close contact and are metabolically coupled (Kanow et al., 2017); (Adijanto and Philp, 2012), we speculate that the interactions with photoreceptors may regulate the mRNA expression of glucose and monocarboxylate transporters in the RPE.

To test this hypothesis, we first synchronized ARPE-19 monolayers by serum-shock treatment $(t=0-$ $2 \mathrm{~h}$ ) and gave them serum free medium $(\mathrm{t}=2 \mathrm{~h})$. We then incubated cells with POS or medium at time points $t=9-12 h ; t=15-18 h ; t=21-24 h ; t=27-30 h ; t=33-36 h$, harvested the cells and performed RT-PCR analysis.

A 2-way ANOVA analysis showed a treatment effect on SLC2A1 (Fig. 5a, $F(1,20)=5.74, p<0.01$ ), but not on SLC16A1 (Fig. 5b, $F(1,20)=0.49, p=0.49$ ) mRNA expression levels. Time was a significant factor for SLC2A1 (Fig. 5a, F(4, 20) = 5.74, p < 0.01), but not for SLC16A1 (Fig. 5b, $F(4,20)=0.86, p=0.51$ ) mRNA expression levels. Time $x$ treatment was significant for SLC2A1 (Fig. 5a, $F(4,20)=4.33, p<0.05$ ) and had a tendency for SLC16A1 (Fig. 5b, $F(4,20)=2.56, p=0.07$ ) mRNA expression levels. Further, Holm-Sidak's post-hoc test revealed that POS incubation performed at $t=27-30 h$, significantly decreased SLC2A1 $(p<0.001)$ and SLC16A1 $(p<0.05)$ mRNA expression. In contrast, we did not observe any effects of a $3 \mathrm{~h}$ or $6 \mathrm{~h}$ POS incubation on transporter gene expression in non-synchronized ARPE-19 monolayers (Fig. S1). These results suggest that POS can exert a phase-dependent effect on gene expression of glucose and monocarboxylate transporters in the RPE. 

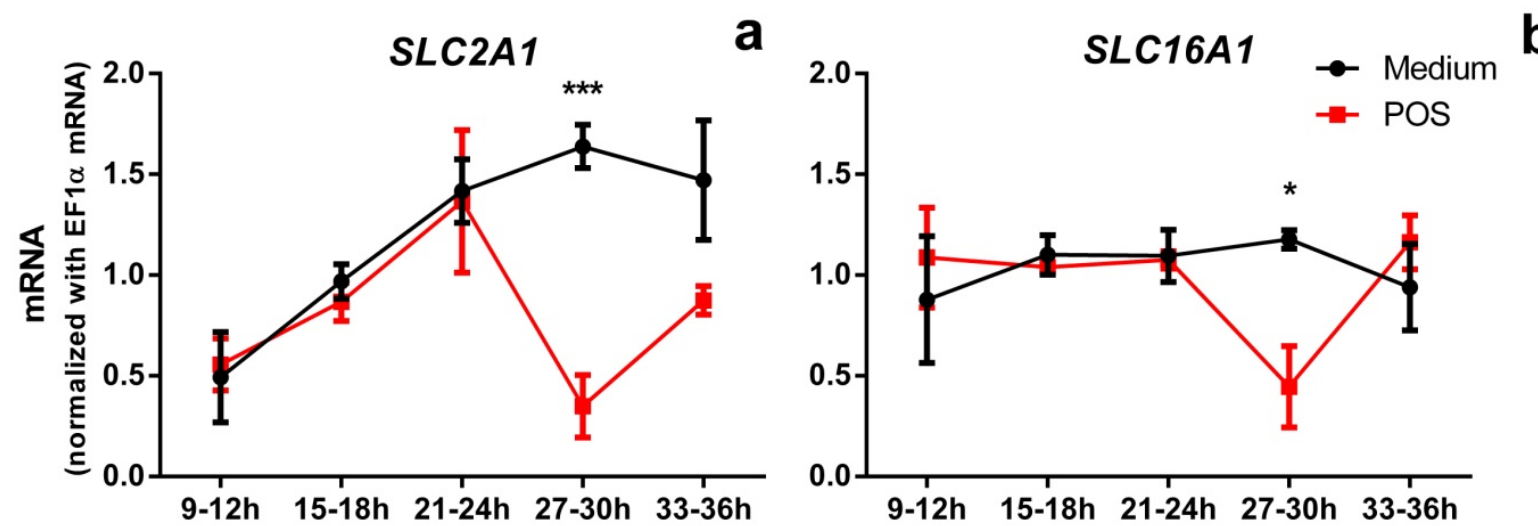

382

383

384

385

386

387

388

389

390

391

392

393

394

395

396

397

398

399

400

401

402

403

404

405

Figure 5. POS incubation has a phase dependent effect on transporter gene expression in ARPE-19 monolayers. (a, b) Cells were synchronized using medium containing $50 \%$ serum for $2 \mathrm{~h}(\mathrm{t}=0-2 \mathrm{~h})$ and received serum-free medium at $t=2 \mathrm{~h}$. Cells were incubated with POS (red lines) either between $\mathrm{t}=9$ $12 \mathrm{~h}, \mathrm{t}=15-18 \mathrm{~h}, \mathrm{t}=21-24 \mathrm{~h}, \mathrm{t}=27-30 \mathrm{~h}$ or $\mathrm{t}=33-36 \mathrm{~h}$. Incubation with medium (black lines) at the same time-points was used as a control. At $t=27-30 \mathrm{~h}$ POS significantly down-regulated the mRNA expression of SLC2A1 (a, Holm-Sidak's post-hoc test, $\mathrm{p}<0.001$ ) and SLC16A1 (b, Holm-Sidak's post-hoc test, $\mathrm{p}<$ $0.05)$. Values are plotted as means \pm SEM, $n=3$ / time-point / treatment. ${ }^{*} p<0.05,{ }^{* * *} p<0.001$

\section{Discussion}

In this study, we tested the hypothesis that the RPE clock regulates RPE-mediated glucose and lactate transport. We report rhythmic mRNA expression of the most abundant glucose (SLC2A1) and monocarboxylate (SLC16A1) transporters in ARPE-19 monolayers. In these cells, time did not affect GLUT1 protein levels, in contrast to MCT1 proteins which did vary over time. Our results suggest that rhythmic expression of these transporters is influenced by RPE-intercellular coupling and interactions with photoreceptors via POS. Finally, our results suggest that RPE-mediated lactate transport is rhythmic, but we found no evidence of rhythmicity of RPE-mediated glucose transport.

We first characterized the glucose (SLC2A) and monocarboxylate (SLC16A) transporter gene expression profile in a human RPE cell line (ARPE-19). We found that SLC2A1 was the most abundant glucose transporter in ARPE-19. This finding is in agreement with previously published work using fresh RPE cultures (Senanayake et al., 2006), laser capture microdissected RPE from human donor eyes (van 
Soest et al., 2007) and relevant data from mouse RPE (analysis of the GEO data set GSE10246 (Lattin et al., 2008) performed by Swarup et al., 2019). We report apical localization of the protein product of SLC2A1, GLUT1 in our ARPE-19 monolayers corroborating previous results (Senanayake et al., 2006), but lacking basolateral expression which is an in vivo characteristic (Philp et al., 2003a; Philp et al., 2003b; Sugasawa et al., 1994; Swarup et al., 2019). The uptake of glucose by monolayer cultures from apical and basolateral supernatants was similar. Thus, it is possible that basolateral glucose might be ingested by other transporters which were not studied in this paper.

The monocarboxylate transporter SLC16A1 was the highest expressed lactate transporter in our ARPE19 cultures. We localized the corresponding protein, MCT1, to the apical RPE membrane of monolayers. These findings are also in agreement with the literature (Philp et al., 2003a b). However, in contrast to the findings of Philp and coworkers, we were not able to show SLC16A8 (encoding MCT3) or SLC16A4 (encoding MCT4) mRNA expression in our cultures (Philp et al., 2003a). This inconsistency may be due to differences in culturing conditions as Philp and coworkers cultured ARPE-19 for an extended period (up to 270 days) without using Matrigel coated transwell inserts (Philp et al., 2003b). Our data suggests that cultures secreted lactate in the apical supernatants, suggesting that the MCT1 transporter plays a prominent role in lactate trafficking in our cultures. The gradual increase in basolateral lactate concentrations might be mediated via other MCT transporters expressed by our cultures.

Our study is the first to show rhythmic gene expression of SLC2A1 and SLC16A1 in the RPE. This result is expected since the rhythmic expression of these genes was demonstrated in various tissues in previous studies. For instance, studies have shown rhythmic mRNA expression of S/c2a1 in rat cerebellum (Soltesova et al., 2013) and in numerous baboon organs and tissues including: the brain, bladder, thyroid, duodenum, kidney medulla and omental fat (Mure et al., 2018). In addition, a timedependent regulation of glucose uptake was suggested in ARPE-19 cells (Kim et al., 2007). Likewise, the S/c16a1 mRNA is rhythmically expressed in rat jejunal mucosa (Stearns et al., 2008) and baboon bladder, preoptic area, stomach fundus, omental, retroperitoneal and perirenal fat (Mure et al., 2018). However, the necessity of a monolayer RPE cellular organization for promoting rhythmic gene expression of SLC2A1 and SLC16A1 is not expected. Our recent work showed that the monolayer cellular organization of the RPE enhances the amplitudes and shortens the periods of clock gene oscillations, but is otherwise dispensable for the rhythmicity of these cells (Milićević et al., 2019). It is known that in RPE monolayers cells communicate via gap junctions (Ning et al., 2013; Pearson et al., 2004; Vainio et al., 2015) through which secondary messengers such as $\mathrm{Ca}^{2+}$ and cAMP can freely permeate (Geletu et al., 2012). It may be possible that the rhythmicity of SLC2A1 and SLC16A1 
440 transporters is driven by a secondary messenger-triggered molecular cascade. However, this 441 rhythmicity is translated only for MCT1 protein and not GLUT1 protein. Inconsistencies between mRNA 442 and protein oscillations were reported in literature, which may be explained by differences in degradation kinetics of studied proteins (Jang et al., 2015; Mauvoisin et al., 2014). Further studies are necessary to elucidate these unexpected effects of culturing conditions on transporter mRNA and protein expression.

We report discrepancies in RT-PCR results between our ARPE-19 monolayer samples obtained at $3 \mathrm{~h}$ intervals for 54h (Fig. 2) and the control group in our phase-dependent intervention experiments (Fig. 5). In our previous work we also observed similar inconsistencies (Milićević et al., 2019). It is wellknown that a medium change affects the results of bioluminescence recordings of explant cultures obtained from Per2 ${ }^{L U C}$ transgenic mice (Baba et al., 2015; Guenthner et al., 2014). Thus, the inconsistencies observed between Fig. 2 and 5 in this study are likely due to a phase-dependent effect of medium administration.

We hypothesized that rhythmic regulation of these transporters in the RPE is necessary to meet the time-dependent metabolic needs of the retina. In agreement with this view, we observed in our cultures, that the acrophases of mRNA expression of SLC16A1 (18.59 $\pm 1.78 \mathrm{~h})$ and SLC2A1 (15.94 \pm $1.46 \mathrm{~h}$ ) were similar. The expression of these transporters was affected by POS at the same time-point after synchronization ( $t=27-30 \mathrm{~h}$ ), suggesting that interactions with photoreceptors regulate RPEmediated metabolite transport. Further, lactate concentrations oscillated in apical supernatants. In addition, the concentration gradients of glucose and lactate correlated with the mRNA expression of respective transporters. However, we did not observe significant differences between apical and basolateral glucose concentrations, and no rhythm of glucose concentrations in supernatants. Thus, results from our in vitro assay suggest that the rhythmic regulation of SLC16A1 is reflected on a functional level, whereas we find no evidence for rhythmic glucose transport.

Previous research reported that inhibition of monocarboxylate transport disrupts retinal function, in particular b-wave and oscillatory potential amplitudes in rats (Bui et al., 2004). Considering that we have shown rhythmic regulation of the SLC16A1 gene (encoding MCT1) in the RPE, we speculate that the rhythmicity of this gene plays a role in maintaining retinal health. For instance, during aging nearly all aspects of clock-regulated physiological processes deteriorate. Dysregulation of circadian clocks is considered as a potential mechanism that underlies age-associated changes in physiology and behavior

473 (Hood and Amir, 2017). Accordingly, retinal and RPE clock alterations were reported in aged mice (Baba 
and Tosini, 2018). In addition, clock disruption was shown to exacerbate age-related retinal cell death in mice (Baba et al., 2018). It is therefore, reasonable to speculate that aging disrupts clock regulated metabolite transport, thus accelerating retinal cell death.

\section{Conclusion}

In conclusion, our work provides evidence that RPE-mediated transport of lactate is regulated by the circadian clock. This finding broadens the scope of physiological functions regulated by the circadian clock in the RPE-photoreceptor functional unit and its potential role in maintaining retinal health.

\section{Acknowledgements}

We thank Hella Aberson and Angelica Duursma for technical assistance. This project has been funded with support from the NeuroTime Erasmus+ of the European Commission, RSB Rotterdam, Nelly Reef foundation, Foundation Ooglijders and Het Lot SVLB.

\section{Author Contributions}

NM designed, performed experiments, analyzed data, wrote the manuscript; J.t.B and A.t.A. provided technical assistance; A.B. and M.P.F.S. designed, analyzed and wrote the manuscript.

\section{Competing interests statement}

The authors declare no potential competing interests with respect to the research, authorship, and/or publication of this article.

\section{References}

Adijanto, J., Philp, N.J., 2012. The SLC16A family of monocarboxylate transporters (MCTs)--physiology and function in cellular metabolism, pH homeostasis, and fluid transport. Curr Top Membr 70, 275311.

Ames, A., 3rd, Li, Y.Y., Heher, E.C., Kimble, C.R., 1992. Energy metabolism of rabbit retina as related to function: high cost of $\mathrm{Na}+$ transport. The Journal of neuroscience : the official journal of the Society for Neuroscience 12, 840-853. 
509 Baba, K., Davidson, A.J., Tosini, G., 2015. Melatonin Entrains PER2::LUC Bioluminescence Circadian

510 Rhythm in the Mouse Cornea. Investigative ophthalmology \& visual science 56, 4753-4758.

511 Baba, K., DeBruyne, J.P., Tosini, G., 2017. Dopamine 2 Receptor Activation Entrains Circadian Clocks 512 in Mouse Retinal Pigment Epithelium. Scientific reports 7, 5103.

513 Baba, K., Piano, I., Lyuboslavsky, P., Chrenek, M.A., Sellers, J.T., Zhang, S., Gargini, C., He, L., Tosini, G., 514 luvone, P.M., 2018. Removal of clock gene Bmal1 from the retina affects retinal development and 515 accelerates cone photoreceptor degeneration during aging. Proceedings of the National Academy of 516 Sciences of the United States of America 115, 13099-13104.

517 Baba, K., Sengupta, A., Tosini, M., Contreras-Alcantara, S., Tosini, G., 2010. Circadian regulation of the 518 PERIOD 2::LUCIFERASE bioluminescence rhythm in the mouse retinal pigment epithelium-choroid. 519 Molecular vision 16, 2605-2611.

520 Baba, K., Tosini, G., 2018. Aging Alters Circadian Rhythms in the Mouse Eye. Journal of biological 521 rhythms 33, 441-445.

Balsalobre, A., Damiola, F., Schibler, U., 1998. A serum shock induces circadian gene expression in mammalian tissue culture cells. Cell 93, 929-937.

524 Bui, B.V., Kalloniatis, M., Vingrys, A.J., 2004. Retinal function loss after monocarboxylate transport

525 inhibition. Investigative ophthalmology \& visual science 45, 584-593.

526 Gal, A., Li, Y., Thompson, D.A., Weir, J., Orth, U., Jacobson, S.G., Apfelstedt-Sylla, E., Vollrath, D., 527 2000. Mutations in MERTK, the human orthologue of the RCS rat retinal dystrophy gene, cause 528 retinitis pigmentosa. Nature genetics 26, 270-271.

529 Geletu, M., Trotman-Grant, A., Raptis, L., 2012. Mind the gap; regulation of gap junctional, 530 intercellular communication by the SRC oncogene product and its effectors. Anticancer research 32 , $5314245-4250$.

532 Guenthner, C.J., Luitje, M.E., Pyle, L.A., Molyneux, P.C., Yu, J.K., Li, A.S., Leise, T.L., Harrington, M.E., 533 2014. Circadian rhythms of Per2::Luc in individual primary mouse hepatocytes and cultures. PloS one 5349 , e87573.

535 Harris, V.M., 2015. Protein detection by Simple Western analysis. Methods in molecular biology 536 (Clifton, N.J.) 1312, 465-468.

537 Hood, S., Amir, S., 2017. The aging clock: circadian rhythms and later life. The Journal of clinical 538 investigation $127,437-446$.

539 Ikarashi, R., Akechi, H., Kanda, Y., Ahmad, A., Takeuchi, K., Morioka, E., Sugiyama, T., Ebisawa, T., 540 Ikeda, M., Ikeda, M., 2017. Regulation of molecular clock oscillations and phagocytic activity via 541 muscarinic $\mathrm{Ca}(2+)$ signaling in human retinal pigment epithelial cells. Scientific reports 7, 44175. 
Jang, C., Lahens, N.F., Hogenesch, J.B., Sehgal, A., 2015. Ribosome profiling reveals an important role

543 for translational control in circadian gene expression. Genome research 25, 1836-1847.

544 Kanow, M.A., Giarmarco, M.M., Jankowski, C.S., Tsantilas, K., Engel, A.L., Du, J., Linton, J.D., 545 Farnsworth, C.C., Sloat, S.R., Rountree, A., Sweet, I.R., Lindsay, K.J., Parker, E.D., Brockerhoff, S.E., 546 Sadilek, M., Chao, J.R., Hurley, J.B., 2017. Biochemical adaptations of the retina and retinal pigment 547 epithelium support a metabolic ecosystem in the vertebrate eye. elife 6.

548 Kim, D.I., Lim, S.K., Park, M.J., Han, H.J., Kim, G.Y., Park, S.H., 2007. The involvement of 549 phosphatidylinositol 3-kinase /Akt signaling in high glucose-induced downregulation of GLUT-1 550 expression in ARPE cells. Life sciences 80, 626-632.

551 Lattin, J.E., Schroder, K., Su, A.I., Walker, J.R., Zhang, J., Wiltshire, T., Saijo, K., Glass, C.K., Hume, D.A., 552 Kellie, S., Sweet, M.J., 2008. Expression analysis of G Protein-Coupled Receptors in mouse 553 macrophages. Immunome research 4, 5.

554 Lin, M.K., Kim, S.H., Zhang, L., Tsai, Y.T., Tsang, S.H., 2015. Rod metabolic demand drives progression 555 in retinopathies. Taiwan journal of ophthalmology 5, 105-108.

556 Mauvoisin, D., Wang, J., Jouffe, C., Martin, E., Atger, F., Waridel, P., Quadroni, M., Gachon, F., Naef, 557 F., 2014. Circadian clock-dependent and -independent rhythmic proteomes implement distinct 558 diurnal functions in mouse liver. Proceedings of the National Academy of Sciences of the United 559 States of America 111, 167-172.

560 McMahon, D.G., luvone, P.M., Tosini, G., 2014. Circadian organization of the mammalian retina: from 561 gene regulation to physiology and diseases. Progress in retinal and eye research 39, 58-76.

562 Milićević, N., Mazzaro, N., de Bruin, I., Wils, E., ten Brink, J., Asbroek, A.t., Mendoza, J., Bergen, A., 563 Felder-Schmittbuhl, M.-P., 2019. Rev-Erba and Photoreceptor Outer Segments modulate the 564 Circadian Clock in Retinal Pigment Epithelial Cells. Scientific reports 9, 11790.

565 Morioka, E., Kanda, Y., Koizumi, H., Miyamoto, T., Ikeda, M., 2018. Histamine Regulates Molecular 566 Clock Oscillations in Human Retinal Pigment Epithelial Cells via H1 Receptors. Frontiers in 567 endocrinology 9, 108.

568 Mure, L.S., Le, H.D., Benegiamo, G., Chang, M.W., Rios, L., Jillani, N., Ngotho, M., Kariuki, T., Dkhissi569 Benyahya, O., Cooper, H.M., Panda, S., 2018. Diurnal transcriptome atlas of a primate across major 570 neural and peripheral tissues. Science (New York, N.Y.) 359.

571 Narayan, D.S., Chidlow, G., Wood, J.P., Casson, R.J., 2017. Glucose metabolism in mammalian 572 photoreceptor inner and outer segments. Clinical \& experimental ophthalmology 45, 730-741.

573 Ng, S.K., Wood, J.P., Chidlow, G., Han, G., Kittipassorn, T., Peet, D.J., Casson, R.J., 2015. Cancer-like 574 metabolism of the mammalian retina. Clinical \& experimental ophthalmology 43, 367-376.

575 Ning, N., Wen, Y., Li, Y., Li, J., 2013. Meclofenamic acid blocks the gap junction communication 576 between the retinal pigment epithelial cells. Human \& experimental toxicology 32, 1164-1169. 
577 Pavan, B., Frigato, E., Pozzati, S., Prasad, P.D., Bertolucci, C., Biondi, C., 2006. Circadian clocks 578 regulate adenylyl cyclase activity rhythms in human RPE cells. Biochemical and biophysical research 579 communications 350, 169-173.

580 Pearson, R.A., Catsicas, M., Becker, D.L., Bayley, P., Luneborg, N.L., Mobbs, P., 2004. Ca(2+) signalling 581 and gap junction coupling within and between pigment epithelium and neural retina in the 582 developing chick. The European journal of neuroscience 19, 2435-2445.

583 Philp, N.J., Ochrietor, J.D., Rudoy, C., Muramatsu, T., Linser, P.J., 2003a. Loss of MCT1, MCT3, and 584 MCT4 expression in the retinal pigment epithelium and neural retina of the 5A11/basigin-null mouse. 585 Investigative ophthalmology \& visual science 44, 1305-1311.

586 Philp, N.J., Wang, D., Yoon, H., Hjelmeland, L.M., 2003b. Polarized expression of monocarboxylate 587 transporters in human retinal pigment epithelium and ARPE-19 cells. Investigative ophthalmology \& 588 visual science 44, 1716-1721.

589 Philp, N.J., Yoon, H., Grollman, E.F., 1998. Monocarboxylate transporter MCT1 is located in the apical 590 membrane and MCT3 in the basal membrane of rat RPE. The American journal of physiology 274, 591 R1824-1828.

592 Senanayake, P., Calabro, A., Hu, J.G., Bonilha, V.L., Darr, A., Bok, D., Hollyfield, J.G., 2006. Glucose 593 utilization by the retinal pigment epithelium: evidence for rapid uptake and storage in glycogen, 594 followed by glycogen utilization. Experimental eye research 83, 235-246.

595 Soltesova, D., Vesela, A., Mravec, B., Herichova, I., 2013. Daily profile of glut1 and glut4 expression in 596 tissues inside and outside the blood-brain barrier in control and streptozotocin-treated rats. Physiological research 62 Suppl 1, S115-124.

598 Stearns, A.T., Balakrishnan, A., Rhoads, D.B., Ashley, S.W., Tavakkolizadeh, A., 2008. Diurnal rhythmicity in the transcription of jejunal drug transporters. Journal of pharmacological sciences 108 , 144-148.

601 Sugasawa, K., Deguchi, J., Okami, T., Yamamoto, A., Omori, K., Uyama, M., Tashiro, Y., 1994. 602 Immunocytochemical analyses of distributions of Na, K-ATPase and GLUT1, insulin and transferrin 603 receptors in the developing retinal pigment epithelial cells. Cell structure and function 19, 21-28.

604 Swarup, A., Samuels, I.S., Bell, B.A., Han, J.Y.S., Du, J., Massenzio, E., Abel, E.D., Boesze-Battaglia, K., 605 Peachey, N.S., Philp, N.J., 2019. Modulating GLUT1 expression in retinal pigment epithelium 606 decreases glucose levels in the retina: impact on photoreceptors and Muller glial cells. American 607 journal of physiology. Cell physiology 316, C121-c133.

608 Tosini, G., Davidson, A.J., Fukuhara, C., Kasamatsu, M., Castanon-Cervantes, O., 2007. Localization of 609 a circadian clock in mammalian photoreceptors. FASEB journal : official publication of the Federation 610 of American Societies for Experimental Biology 21, 3866-3871.

611 Vainio, I., Abu Khamidakh, A., Paci, M., Skottman, H., Juuti-Uusitalo, K., Hyttinen, J., Nymark, S., 2015. 612 Computational Model of Ca2+ Wave Propagation in Human Retinal Pigment Epithelial ARPE-19 Cells. 613 PloS one 10, e0128434. 
614 van Soest, S.S., de Wit, G.M., Essing, A.H., ten Brink, J.B., Kamphuis, W., de Jong, P.T., Bergen, A.A., 615 2007. Comparison of human retinal pigment epithelium gene expression in macula and periphery

616 highlights potential topographic differences in Bruch's membrane. Molecular vision 13, 1608-1617.

617 Yoshikawa, A., Shimada, H., Numazawa, K., Sasaki, T., Ikeda, M., Kawashima, M., Kato, N., Tokunaga, 618 K., Ebisawa, T., 2008. Establishment of human cell lines showing circadian rhythms of 619 bioluminescence. Neuroscience letters 446, 40-44.

620 Yu, D.Y., Cringle, S.J., 2001. Oxygen distribution and consumption within the retina in vascularised 621 and avascular retinas and in animal models of retinal disease. Progress in retinal and eye research 20, $622 \quad 175-208$. 